\title{
SPOKEN LANGUAGE IDENTIFICATION USING LARGE VOCABULARY SPEECH RECOGNITION.
}

\author{
James L Hieronymus and Shubha Kadambe* \\ Bell Laboratories, 700 Mountain Avenue, Murray Hill, NJ 07974 \\ Atlantic Aerospace Elect. Corp., 6404 Ivy Lane,Greenbelt, MD 20906*
}

\begin{abstract}
A task independent spoken Language Identification (LID) system which uses a Large Vocabulary Automatic Speech Recognition (LVASR) module for each language to choose the most likely language spoken is described in detail. The system has been trained on 5 languages: English, German, Japanese, Mandarin Chinese and Spanish. In this paper it is demonstrated that the performance of a LID system which is based on LVASR gives very good performance, when trained and tested on a 5 language subset (English, German, Spanish, Japanese, and Mandarin Chinese) of the Oregon Graduate Institute 11 language data base. The performance advantage is shown for both long (50 second) and short ( 10 second) test utterances. The five language results show $88 \%$ correct recognition for 50 second utterances without confidence measures and $98 \%$ correct with confidence measures. The recognition rate is $81 \%$ correct for 10 second utterances without confidence measures and $93 \%$ correct with confidence measures. The best performance has been obtained for systems trained on phonetically hand labeled speech.
\end{abstract}

\section{INTRODUCTION}

In the future, Language Identification (LID) systems will be an integral part of telephone and speech input computer networks which provide services in many languages. A LID system can be used to pre-sort the callers into the language they speak, so that the required service will be provided in the language appropriate to the talker. Examples of these services include, travel information, emergency assistance, language interpretation, telephone information, buying services, banking and stock trading. Since there are large numbers of non-English talkers in the US population due to immigration, there is a need to offer multi-language capability even domestically. International tourism adds to the desirability of offering services in many language.

Language identification has been the subject of research for many years. Initially, systems were developed to screen radio transmissions and telephone conversations for the intelligence community. The systems developed were useful in screening transmissions for a few target languages which were subsequently monitored by humans.

The languages of the world differ from one another along many dimensions which have been codified as linguistic cat- egories. These include, phoneme inventory, phoneme sequences, syllable structure, prosodics, lexical words and grammar. Therefore, we hypothesize that an LID system which exploits each of these linguistic categories in turn will have the necessary discriminative power to provide good performance on short utterances.

This paper is divided into five sections. First, past work will be discussed and its relevance to the present study noted. Second, the basic phoneme recognition system is described. Third, the architecture of the LVASR system is discussed. Fourth, details of training the system are given, this involved selecting a vocabulary and constructing a word sequence model for each language. Finally, the LID results for the system using LVASR sub-systems are discussed. These show that the more complete language modeling which the LVASR system provides gives the best performance.

\section{PAST WORK}

Neuburg and House [2] used an ergodic Markov model of sequences of 5 broad phonetic categories (stop consonant, fricative consonant, non-vocalic sonorant, vowel and silence to identify 8 languages with $100 \%$ accuracy using phonetic hand labels for input, when they tested on the training data (because of the scarcity of data for the experiment). The system was designed to have a variable number of states (from 2 to 5 ) to model each language. A version of our system also gets $100 \%$ correct language identification using automatically obtained fine phonetic labels and a trigram phonemotactic model (corresponding to a 3 state model in the Neuburg and House system) for five languages when tested on training data.

Recently, there has been much interest in phonetic modeling of speech for language identification. Muthusamy et al developed a language identification system based on broad phonetic classes and neural network classifiers [3]. Muthusamy [4] also collected an 11 language telephone speech database at Oregon Graduate Institute which became the standard training and testing database for a series of U.S. Government sponsored language identification tests which were administered by the National Institute for Standards and Technology (NIST). These tests provide a way of measuring the relative performance of many systems incorporating different methods of spoken language identification. The results reported in this paper are obtained from the Spring 1994 training and test data for these tests. 
In the past three years, a number of researchers $[5,6,7]$ have been developing systems which first recognize phonemes using HMM phoneme modeling and then use a phonemotactic model of phoneme sequences allowed within each language to identify the spoken language. Our baseline system described in [7] uses Continuous Density second order Variable Duration Hidden Markov Model (CVDHMM) to achieve the phoneme recognition based on context conditioned phonemes (called tri-phones in the literature) and trigram phoneme sequence models (phonemotactic models). The other LID systems [6,5] mentioned above, use context independent first order Markov models for phoneme recognition with bigram phonemotactic models. For languages with Consonant-Vowel-Consonant (CVC) syllable structure, the trigram models do a very good job of modeling the most frequent words, which are usually monosyllabic and hence, should help in discriminating these languages more efficiently than bigram phonemotactic models. On the other hand languages with Consonant-Vowel syllable structure would be more efficiently modeled by the bigram word models. The languages studied represented a mixture of syllable structures, so that the trigram word models seem to provide an advantage.

Language identification systems have been developed which use varying degrees of linguistic knowledge[8]. The question remains about how much linguistic knowledge is necessary to get the desired performance, given that adding linguistic knowledge into a system is rather labor intensive. This paper shows that full sentence recognition leads to better performance, than the same system using trigram phonotactics, and language specific phonemes alone. Developing automated methods for creating large vocabulary speech recognition systems for new languages would allow these performance advantages to be realized for language identification without extensive human effort.

\section{DESCRIPTION OF THE PHONEME RECOGNITION SYSTEM}

The phoneme recognition system was developed at Bell Labs for English by A. Ljolje [1]. This phoneme recognizer is based on a second order ergodic CVDHMM. The ergodic HMM has one state per phoneme. However, the acoustic model for each phoneme is a time sequence of three probability density functions (pdf's) with each pdf representing the beginning, the middle and the end of a phoneme, respectively. The pdf's are represented as mixtures of Gaussian pdf's on the acoustic features (Cepstral coefficients from LPC analysis of the speech waveform) which have been decorrelated. This structure is equivalent to a three state left-to-right HMM phoneme model. The duration of each phoneme is modeled by a four parameter gamma distribution function. The four parameters are: (1) the shortest allowed phoneme duration (the gamma distribution shift), (2) the mean duration, (3) the variance of the duration, and (4) the maximum allowed duration for the phoneme. The shortest allowed duration is equal to the shortest observed duration in the training data, the mean and variance are calculated from the training data and the maximum duration is calculated as the $95^{t h}$ percentile of the distribution. Because the ergodic HMM is second order, the transition probabilities are the probability of the next phoneme given the past two phonemes. This is then the trigram phoneme sequence probability which can be estimated separately. A diagram of a Second Order Ergodic HMM is shown in Figure 1.

\section{First Phoneme Second Phoneme Third Phoneme}

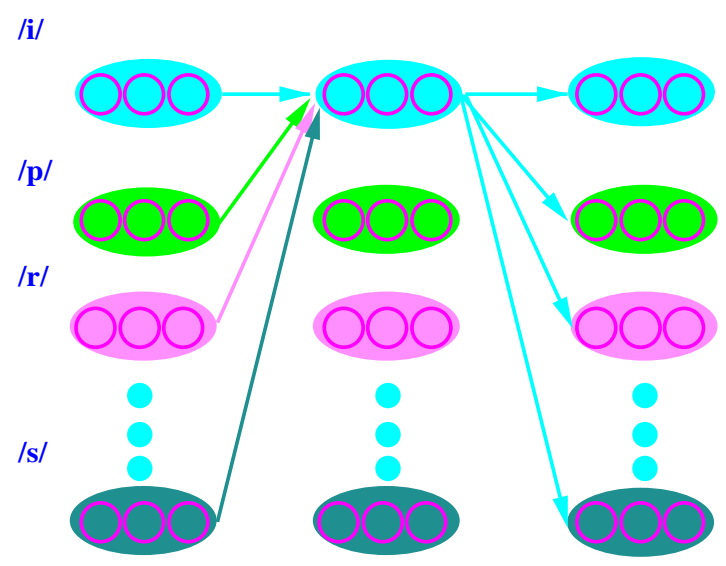

Figure 1. The architecture of a second order ergodic CVDHMM

\subsubsection{Acoustic features}

The speech feature vector consists of 26 coefficients which were chosen from 38 coefficients of 12 cepstra, 12 delta cepstra, 12 delta delta cepstra, delta energy and delta delta energy using a discriminant analysis method [9] First all the cepstral coefficients are computed using an autocorrelation LPC model with a 20 msec time window which is shifted by $10 \mathrm{msec}$ per frame. Then the coefficients are decorrelated (rotated to be orthogonal). The decorrelation is most needed for the cepstral and delta delta cepstral coefficients.

\subsubsection{Training phoneme models}

Different training procedures were adopted to train the phoneme recognizer, described above, depending on the type of transcription available for each language. (1) When words and their start and end times were available, phonemes and average durations were obtained from a Text to Speech (TTS) system, and linearly warped to the word duration. The derived phoneme segments thus obtained are used to train preliminary HHM models. These models are used to re-segment the data. The models are re-trained iteratively using a segmental k-means algorithm until the models converge. (2) When a time aligned phonemic transcription of the speech data is available, the initial models are trained using this data and the models are re-trained using the segmental k-means algorithm iteratively until the models converge. (3) When the sentence level transcription and segmentation is available, the phonemic level transcription and segmentation is obtained automatically as described in method 1 except that the phoneme durations are stretched linearly to fit the whole sentence. The models are trained iteratively as described in method 1 by using the segmented data so obtained. The phonemic boundaries obtained by 
this procedure are less reliable than the ones obtained from the hand labels; however, the system converges to stable models. Results are best for method 2 .

\section{LID SYSTEM USING LEXICAL ACCESS WITH GRAMMAR}

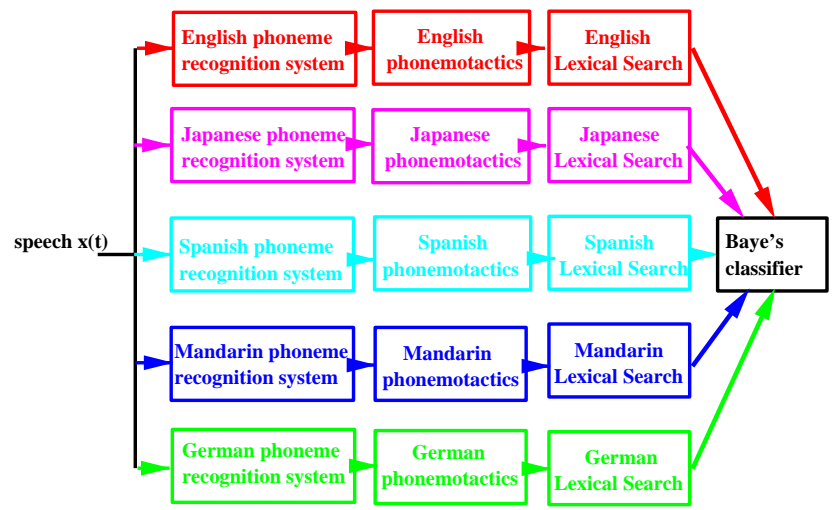

Figure 2. The block diagram of the complete LID system

A full LVASR system is used for language identification. The block diagram of the LID system is as shown in Figure 2.

The lexical access system uses cascades of weighted finite state transducers [10] to do lexical search and grammatical constraints. The first step is a transduction from phoneme lattices to word lattices. The best path through the finite state network provides the most probable word sequence and the probability, for words in the vocabulary without a word sequence model. The second transduction is from a word lattice to a sentence lattice, which obeys the bigram word grammar trained on the OGI training and development test portions of the corpus. The best path though the resulting sentence lattice is the most probable sentence given the language model. For language identification, the subsystems (block 1, 2 and 3 in Figure 2) for each language are run in parallel for a given speech signal similar to the base line system described above. The language subsystem with the highest normalized log likelihood is chosen as the language of the input speech signal.

\section{TRAINING THE LID SYSTEM}

The five language (English, German, Mandarin Chinese, Japanese and Spanish) LID system was trained and tested using the prompted monologue section of the 11 language speech data base collected by OGI [4]. The training and test data consists of about 80 and 18 speakers, respectively, per language. The monologue recording is 50 seconds in length, including pauses, which yields $35-45$ sec of speech.

The acoustic HMM models of English and Spanish phoneme recognition systems were originally trained using method 1 and, the Mandarin, Japanese and German recognition systems were trained using method 2 as described in section 3.0.2. The LID results of training with the orthographically transcribed data is significantly worse than training with phonetically hand lapelled data. Subsequently, the English system was retrained with phonetic hand labels. The performance differences between the two styles of training is discussed in the results section.

\subsection{Training the Word and language model}

For each of the 5 languages, the vocabulary was chosen by taking every unique word from the prompted monologues in the training and devtest portion of the OGI 11 language database. Table 1 below shows the number of words in the lexicon for each language and their average length in phonemes. A bigram language model was trained for each language using the Katz backoff method described in [11]. An attempt was made to add text from newspaper sources to augment the language model, but this resulted in poorer performance, because newspaper style is very different from spoken language.

\begin{tabular}{|l|c|c|}
\hline Language & \# words & average length \\
\hline English & 2564 & 7.47 \\
\hline Spanish & 2014 & 11.36 \\
\hline German & 1844 & 8.34 \\
\hline Japanese & 1863 & 7.80 \\
\hline Mandarin & 1546 & 4.07 \\
\hline
\end{tabular}

Table 1. Lexicon Sizes for Each Language

\subsection{Training the Final Classifier}

The LVASR LID system has many differences in the language models. Different languages have a different number of phonemes, different average word lengths, different word sequences which may contain high frequency words like particles and articles. The result of these differences is that the scores from each subsystem has to be normalized relative to the scores for the other languages. Additive and multiplicative factors have been considered (which for log likelihoods correspond to multiplicative and exponential factors on the probabilities). The additive factors seemed to work best, with the factors trained on the home town section of the OGI corpus.

\section{EXPERIMENTAL DETAILS AND RESULTS}

The LID system was first tested using the training data in order to compare the performance of the present system with the system of Newberg and House discussed above. The system was then tested using the data from the 1994 LID evaluation which was monitored by NIST. The evaluation test data used the full $50 \mathrm{sec}$ recordings and also used short intervals of speech of 10 secs long, with 7210 
sec long utterances per language. These were obtained by segmenting the 50 sec long utterances from each speaker of the test data into 4 segments as specified by NIST. The evaluation data was separate from the data used in training the acoustic and language models. The phonemotactic system recognizes the language spoken $100 \%$ of the time when tested on the training data. When tested on the 1994 evaluation test data, the phonotactic system achieves $88 \%$ correct identification of the language spoken for the $50 \mathrm{sec}$ recordings and $76 \%$ correct for the $10 \mathrm{sec}$ utterances. The performance without the phonemotactic model is considerably worse, averaging $72 \%$ compared with $91 \%$ when the phonemotactic constraint was used on a three language subset for the 50 second utterances.

\begin{tabular}{|l|c|c|c|c|c|}
\hline Length and condition & English & German & Spanish & Japanese & Mandarin \\
\hline $\mathbf{5 0}$ sec no grammar & $85 \%$ & $90 \%$ & $\mathbf{7 6 \%}$ & $\mathbf{9 3} \%$ & $94 \%$ \\
$\mathbf{5 0}$ sec grammar & $\mathbf{9 5} \%$ & $\mathbf{9 5} \%$ & $94 \%$ & $97 \%$ & $97 \%$ \\
$\mathbf{5 0}$ sec normalized & $\mathbf{9 8} \%$ & $\mathbf{9 9} \%$ & $\mathbf{9 6} \%$ & $\mathbf{9 8} \%$ & $\mathbf{9 8} \%$ \\
\hline 10 sec no grammar & $81 \%$ & $82 \%$ & $79 \%$ & $83 \%$ & $81 \%$ \\
10 sec grammar & $85 \%$ & $84 \%$ & $81 \%$ & $87 \%$ & $85 \%$ \\
10 sec normalized & $95 \%$ & $\mathbf{9 6 \%}$ & $90 \%$ & $\mathbf{9 2} \%$ & $90 \%$ \\
\hline
\end{tabular}

Table 2. Five language identification results.

The full large vocabulary LID system was tested on the 1994 evaluation data. The system identified the language spoken an average of $88 \%$ LID rate on five languages on the 50 secs utterances and $81 \%$ on the 10 secs utterances. In Table 2, the results for five languages are shown.

In order to improve the performance of the identification rate for the LVASR system, a technique which involves normalizing the sentence recognition log likelihood, by the log likelihood of the best unconstrained phoneme sequence has been tried. This is similar to the technique used by Young and Ward to detect out of vocabulary words in a large vocabulary ASR system [12]. Later publications refer to this as a confidence measure, and is a measure of how well an unconstrained acoustic model matches the utterance. It was first used for LID by Newman et al. [13]. The equation for the recognition of an utterance in a particular language is

$$
\mathrm{P}\left(\mathrm{S}_{\mathrm{i}}, \mathrm{L}_{\mathrm{i}}, \mathrm{P}_{\mathrm{i}} \mid \mathrm{x}\right)=\mathrm{P}\left(\mathrm{x} \mid \beta_{\mathrm{i}}\right) \mathrm{P}\left(\beta_{\mathrm{i}} \mid \mathrm{W}_{\mathrm{i}}\right) \mathrm{P}\left(\mathrm{S}_{\mathrm{i}} \mid \mathrm{W}_{\mathrm{i}}\right) / \mathrm{P}(\mathrm{x})
$$

where the $P_{\mathrm{s}}$ are probabilities, $x$ is the input speech signal, $\beta_{i}$ is the phoneme sequence, $W_{i}$ is the word sequence, $S_{i}$ is the set of all possible sentences for language $i$ and $L_{i}$ is the phonemotactic model of the language $i$. The term in the numerator is often considered to be the same for all the of sentences recognized, and thus neglected. This is no longer true when we wish to make a comparison between the output of recognizers for different languages. The estimation of this term gives a better estimate of the probabilities and thus better performance when comparing different systems. It may also be possible to allow rejection of languages not in the set trained. The method we used to estimate the probability of the acoustic sequence is to run the phoneme recognizer with equal trigram probability for all possible phoneme sequences. A better estimate might be to compute the best acoustic score based on the best match for all of the Gaussian mixtures in the system. This was the measure used by Newman et al. [13] and will be explored in future work. The present estimate raises the performance of the system to the final best result of $98 \%$ correct identification for $50 \mathrm{sec}$ and $93 \%$ correct identification for $10 \mathrm{sec}$ of speech. The results are shown in Table 2 with the label normalized.

\section{CONCLUSIONS}

A five language identification system based on phonological and lexical models was described. LID results for five languages were reported with the best results being obtained for the full large vocabulary speech recognition system, with a normalization or confidence estimate based on unconstrained phoneme matches for each language. Best results were obtained for a system trained with phonetically hand lapelled data.

\section{ACKNOWLEDGMENTS}

We would like to thank Andrej Ljolje, Mike Riley, Fernando Pereira, Richard Sproat, Bernd Moebius, Chilin Shih and Padma Ramesh for helpful discussions.

\section{REFERENCES}

[1] A. Ljolje, High Accuracy Phone Recognition Using Context Clustering and Quasi-triphonic Models, Computer Speech and Language, to appear.

[2] A. S. House and E. P. Neuberg,(1977) "Toward Automatic Identification of the Language of an Utterance. I. Preliminary Methodological Considerations", J. Acoust. Soc. Am., 62, 708713,1977

[3] Y. K. Muthusamy and R. A. Cole, (1992), "Automatic segmentation and identification of ten languages using telephone speech," Proc of ICSLP 92, Banff, Canada

[4] Y. K. Muthusamy, R. A. Cole and B. T. Oshika, (1992), "The OGI Multi-Language Telephone Speech Corpus", Proc. of ICSLP 92, Banff, Canada.

[5] M. A. Zissman and Elliot Singer,(1994) "Automatic Language Identification of Telephone Speech Messages Using Phoneme Recognition and N-Gram Modeling", Proc of ICASSP 94, Adelaide, Australia, April 1994

[6] L. F. Lamel and J. L. Gauvain, (1994), "Language Identification Using Phone-based Acoustic Likelihoods", Proc. of ICASSP 94, Adelaide, Australia, 1994

[7] S. Kadambe and J. L. Hieronymus, (1994, "Spontaneous speech language identification with a knowledge of linguistics", Proc of ICSLP 94, Yokohama, Japan, pp.

[8] S. Kadambe and J. L. Hieronymus, (1995, "Language Identification with Phonological and Lexical Models", Proc of ICASSP 96, pp. 3507-3510

[9] E. Bocchieri and J. G. Wilpon, (1992), "Discriminative Analysis for Feature Reduction in Automatic Speech Recognition," Proc. of ICASSP 92, Vol. I, pp. 501-504

[10] F. Pereira, M. Riley and R. Sproat, (1994, "Weighted Rational transduction and their application to human language processing", DARPA Workshop on Human Language Tech, Princeton, N.J, 1994

[11] D. Hindle, Preprint 1994

[12] Sheryl Young, (1993), "Detecting Misrecognitions and Out-ofVocabulary Words", Proc of ICASSP 94, Vol. II, pp. 21-24

[13] Sergio Mendoza, Larry Gillick, Yoshiko Ito, Stephen Lowe, and Michael Newman, "Automatic Language Identification Using Large Vocabulary Continuous Speech Recognition," Proc. of ICASSP 96, Vol 2, pp. 785-788. 